\title{
Characterizing the Regional Structural Difference of the Brain between Tau Transgenic (rTg4510) and Wild-Type Mice Using MRI
}

\author{
Zhiyong Xie, Dewen Yang, Diane Stephenson, Daniel Morton, \\ Carol Hicks, Tracy Brown, and Thomas Bocan \\ Pfizer Inc, Groton, CT, United States \\ \{Zhiyong.Xie, Dewen.Yang, Diane.Stephenson, Daniel.Morton, \\ Carol.Hicks, Tracy.Brown, Thomas.Bocan\} @pfizer.com
}

\begin{abstract}
Tg4510 transgenic mouse model demonstrates features resembling Alzheimer's disease including neurofibrillary degeneration and progressive neuronal loss. We investigated the volumetric differences of brain structures between transgenic and wild-type mice using MR images of fourteen 5.5 month old female mice. Tensor-based morphometry and atlas-based segmentation were applied to MRI images. Severe atrophy of hippocampus and neocortex as well as ventricular dilatation were observed in the transgenic mice. These findings were confirmed by histopathologic evaluation of the same mice. The results suggest that MRI should be useful for evaluating disease-modifying therapies for Alzheimer's disease in the rTg4510 model and comparing treatment responses in mice and humans.
\end{abstract}

Keywords: Alzheimer, rTg4510, MRI, Brain, Atlas-based segmentation, Tensor-based morphometry.

\section{Introduction}

Alzheimer's disease is characterized by deposition of neurofibrillary tangles that consist of abnormally hyperphosphorylated tau [1]. Neuronal loss in neocortex and hippocampus are closely associated with the process of neurofibrillary degeneration [2]. Different transgenic mouse models of Alzheimer's neuropathology have been created to advance the understanding of the disease and development of treatments [3]. The rTg4510 mouse model is characterized by conditional overexpression of hyperphosphorylated human P301L mutant tau and profound neurofibrillary pathology, neurodegeneration and behavioral impairment [4]. Spatial memory deficits, progressive increase in neurofibrillary tangles and rapidly progressing neuronal loss have been reported to occur by 5.5 months of age [4].

MRI has been widely investigated as a biomarker to diagnose and estimate the disease progression in Alzheimer's disease [5][6][7]. In this study, we evaluated the sensitivity of in-vivo MRI in characterizing the morphological change of rTg4510 mice at 5.5 months. MR brain images of seven double transgenic rTg4510 female mice and 7 age-matched wild-type female mice were acquired. Tensor-based 
morphometry (TBM) was performed on brain images to inspect regional morphological changes. To quantify the changes in different structures, we segmented the hippocampus, neocortex, ventricle, and cerebellum from MR images of each mouse and compared the size of these structures between the two groups. The results indicated that severe atrophy in the neocortex and hippocampus and dilation of lateral ventricles occurred in transgenic rTg4510 mice while the size of cerebellum remained unchanged. These results were confirmed by histopathologic examination performed in the same mice.

\section{Material and Methods}

\subsection{Animals and MR Imaging}

All animal handling procedures were carried out in compliance with the NIH Guide for the Care and Use of Laboratory Animals under a protocol approved by the Pfizer Global Research and Development Animal Care and Use Committee.

Seven double transgenic rTg4510 female mice and seven age-matched wild-type (WT) female mice (5 months 5 days to 5 months 12 days of age, $22-31 \mathrm{~g}$ ) were evaluated. The mice were housed in a ventilated, temperature-controlled room with a 12hour light/dark cycle.

Brain MRI was performed on a horizontal bore 4.7T magnet (Bruker Biospec 47/40, Bruker-Biospin, Inc). After an initial 5-10 min 2.5\% isoflurane anesthesia induction period, all mice were anesthetized and maintained with $1.6-2.0 \%$ isoflurane in oxygen delivered via a nose cone. The mice were positioned in prone position with heads fixed to the plastic nose cone with the aid of a tooth bar and ear pins. Body temperature was recorded and maintained at 35.5-37.5C using a water heated animal bed and a water heated blanket (on the top). Respiratory rate was monitored continuously using a small animal monitoring and gating system (SA Instrument, Inc. Stony Brook, NY 11790, USA). RF excitation for imaging was delivered through a $72 \mathrm{~mm}$ volume coil, and an actively decoupled mouse brain quadrature surface coil placed on the head was used as the receiver. T2-weighted 3D RARE images were acquired with the following parameters: field of view $=16 \times 16 \times 19.2 \mathrm{~mm} 3$, matrix dimensions $=$ $128 \times 128 \times 64$, spatial resolution $=125 \mu \mathrm{m} \times 125 \mu \mathrm{m} \times 300 \mu \mathrm{m}, \mathrm{TR}=2600 \mathrm{~ms} \mathrm{TE}=$ $23 \mathrm{~ms}$, RARE factor $=16$. The total imaging time was $44 \mathrm{~m} 22 \mathrm{~s} 400 \mathrm{~ms}$.

After MR imaging, mice were euthanized using carbon dioxide gas. The brains were collected, fixed by immersion in $10 \%$ neutral buffered formalin, and embedded in the coronal plane using a mouse brain mold. Each brain produced about 6 slabs $2 \mathrm{~mm}$ thick. Tissues were processed routinely, embedded in paraffin blocks, sectioned at a thickness of $5 \mu \mathrm{m}$, and stained with hematoxylin and eosin (H\&E). All H\&E sections of brain were examined qualitatively by light microscopy.

\subsection{Image Analysis}

The method proposed by Cohen et. al. [8] was used to correct RF inhomogeneity of MR images. Whole brain was segmented from the MR image using an open source software ITK-Snap [9] followed by a manual correction. More specifically, a 3D 
geodesic active contour method [10] implemented in ITK-Snap was used for the semi-automatic brain segmentation.

With the intensity-corrected brain images, we performed tensor-based morphometry [11] to characterize the structural difference between two groups. Besides the TBM study, we also segmented four structures (hippocampus, neocortex, lateral ventricle, and cerebellum) from the MR images and measured the structural size to quantify the difference.

\subsubsection{Tensor-Based Morphometry}

Tensor-based morphometry (TBM) has been widely used to characterize the brain atrophy in Alzheimer's disease in clinical studies [12]. We employed the same method to evaluate the brain morphological differences between transgenic and wildtype mice. We first created a template brain image by registering the brain image of each subject to a pre-selected image, and computing the average of all aligned images. Then we registered all individual brain images to the template, generating a deformation field for each subject. The registration was initialized with a rigid body registration and followed by a nonlinear registration. Rigid body registration was computed by optimizing the mutual information using one-plus-one method [13] implemented in ITK (www.itk.org). Our nonlinear registration method [14], [15] was a splinebased extension to Thirion's Demons technique [16]. It used optical flow to determine the correspondence of voxels which exhibit sufficiently large intensity gradients. Based on the estimated sparse correspondences, a B-spline function of the correspondences over the whole brain volume was determined using weighted scattered data approximation. This two-step algorithm was applied over multiple resolution levels in conventional coarse to fine fashion: both the resolution of the images and the number of spline control parameters were simultaneously adjusted. Specifically, starting with B-spline functions that have a small number of parameters, the algorithm was iterated to match the coarse features of the images. The result was used to initialize the registration at the next resolution level, where the number of spline parameters was increased to allow alignment of the finer features that were apparent in the higher resolution images. This strategy provided a way to incrementally refine the registration and improve the robustness of the method. To correct for variability in intensity, the histograms of the images were matched. The registration method was validated based on the labeled T2 weighted MR images created by RCIBI [16] (http://www.bnl.gov/medical/RCIBI/mouse/), and was capable of yielding overlap ratios of greater than $90 \%$ for big structures (Neocortex, Cerebellum, Thalamus, etc) and $70 \%$ for small structures such as ventricle.

With the deformation field that aligned the brain image of each subject to the template, a 3D Jacobian map can be generated by computing the determinant of local Jacobian matrix at each voxel of template image. The Jacobian determinant was a local measurement of volume difference of each subject relative to the template image. A greater than one value of Jacobian determinant represented voxel expansion during the registration, while a value less than one represented voxel contraction. Since all images were registered to the same template, the Jacobian map can be used to voxel-wise compare the volumetric difference between two groups. To better characterize the local morphological difference, the Jacobian map only included the 
nonlinear deformation. Global transformation such as scaling of the whole brain was not taken into account.

To increase the normality of the data distribution, the Jacobian map for each subject was log-transformed and smoothed with a $0.2 \mathrm{~mm}$ Gaussian kernel. Two tailed ttests were performed at each voxel of smoothed images to find the regions with significant volumetric difference between the two groups.

\subsubsection{Quantification of Size Differences of Different Structures}

To verify the results discovered from the TBM analysis and quantify the volumetric differences between two groups, we measured the volume of anatomical structures in the brain images acquired from each mouse. In both clinical and preclinical studies, hippocampus, neocortex, and lateral ventricle have been used as biomarkers to predict Alzheimer's disease progression [17], [18], [19], [20], [21], [22]. Our TBM study also showed the morphological differences in these regions. Cerebellum was formerly thought to be relatively unaffected in the AD brain. Recent studies revealed that the cerebellum also underwent degenerative changes in Alzheimer's disease [23], [24]. In this study, we segmented hippocampus, neocortex, lateral ventricle, and cerebellum from MRI images of each mouse and compared the size of these structures between groups. The segmentation was done by applying an atlas-based segmentation followed by a manual correction. Based on the T2-weighted MR template and 20 labeled structures from RCIBI [25], we first registered the template to the brain image of each animal, and mapped the label of the template to each subject with computed transformation. Computer-generated segmentation was then reviewed by a trained expert and correction was made if necessary. Fig 1 shows an example of such segmentation.
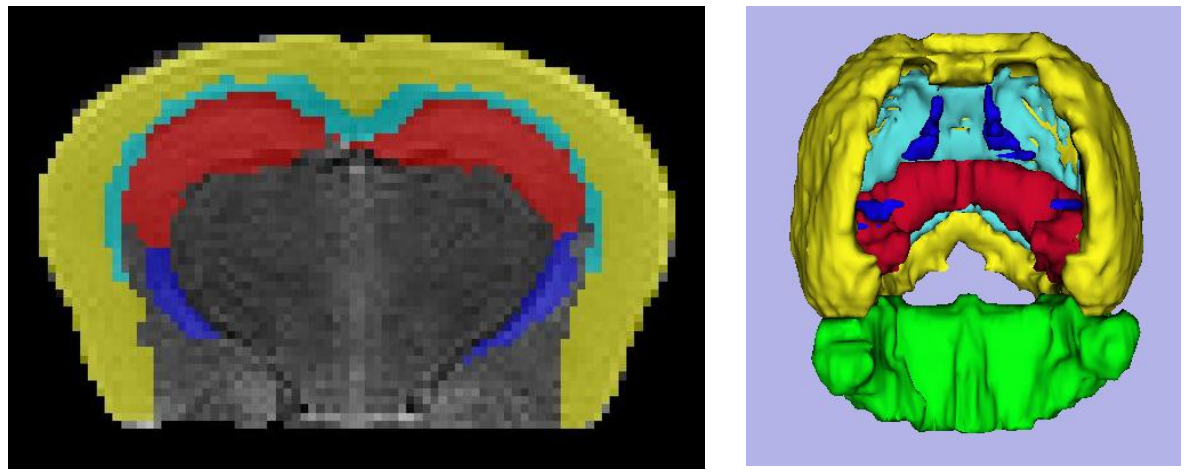

Fig. 1. An example of image segmentation. Left picture shows one slice of a coronal brain image with overlap of colored labels of different structures (yellow $=$ cortex; light blue $=$ corpus callosum \& external capsule; red $=$ hippocampus; dark blue $=$ ventricles; green $=$ cerebellum). 3D visualization of four structures is shown on the right picture.

\section{Results}

The result of TBM analysis showed significant atrophy of neocortex and hippocampus and dilatation of lateral ventricles in the rTg4510 transgenic mice. Figure 2 
demonstrates one slice of a t-score map generated from the voxel-wise two-tailed ttests of Jacobian maps between two groups. The blue color represents regions of volume contraction in transgenic mice and red color indicates the regions of volume expansion. Color bars give the scale of the $\mathrm{t}$-scores. $\mathrm{t}=3.05$ corresponds to $\mathrm{p}=0.01$ for an uncorrected two-tailed t-test.
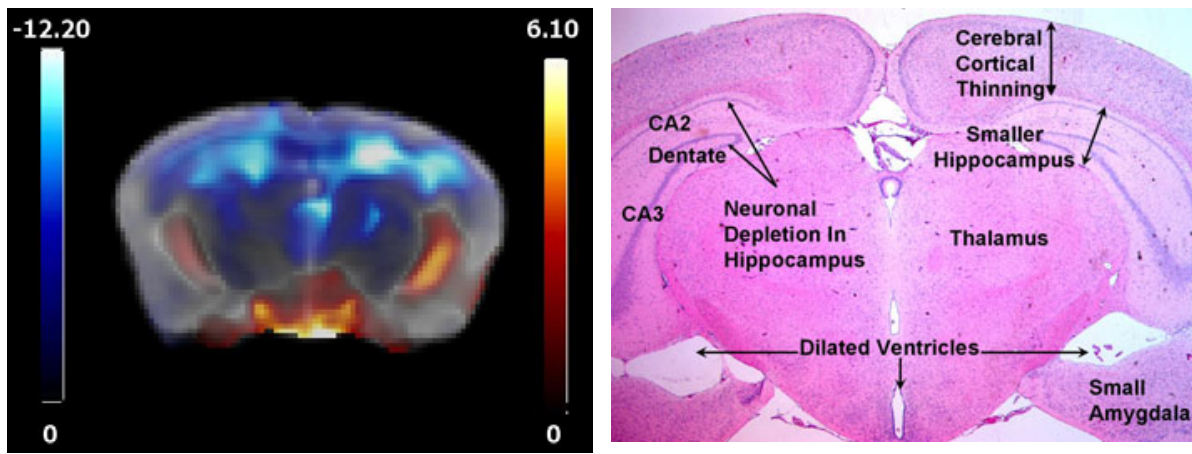

Fig. 2. t-score map of voxel-wise volumetric Fig. 3. A representative histopathology image comparisons between rTg4510 and wild-type of the brain of an $\mathrm{rTg} 4510$ mouse. Thinner mice in a coronal plane at the level of the neocortex, smaller hippocampus and smaller dorsal hippocampus. The blue color represents amygdala were observed. Ventricular dilation regions of structural contraction in transgenic indicated additional loss of brain parenchyma. mice and red color indicates the regions of structural expansion.

Based on the segmentation of hippocampus, neocortex, lateral ventricle, and cerebellum, we computed the volume of different structures for each animal. Figure 4 illustrates the mean volume difference between two groups for hippocampus, neocortex, and lateral ventricle. The hippocampus and neocortex of transgenic mice were significantly smaller than those of wild-type mice ( $\mathrm{p}$ value for two-tailed t-tests were less than 0.0001). Lateral ventricle size of transgenic mice was significantly larger than wild-type mice ( $\mathrm{p}$ value for two-tailed t-test was 0.023 ). No significant difference was observed in the volume of the cerebellum between the two groups.

When we inspected the individual data, we found that all transgenic mice had a smaller hippocampus and neocortex than wild-type mice. Animal to animal variability in $\operatorname{rTg} 4510$ mice was observed in the ventricular volume measurement. 4 out of 7 rTg4510 mice had enlarged lateral ventricles (hydrocephalus) while the other three were similar in size to the wild-type mice. Figure 5 shows one example of a transgenic mouse with enlarged ventricles. The left picture is a slice of a T2-weighted image of the transgenic mouse. The ventricular dilatation (hydrocephalus, shown as bright structures in this picture) is evident in the rTg4510 brain which was not observed in the corresponding slice of a wild-type mouse (right picture, Figure 4). Hydrocephalus likely reflects expansion of the fluid-filled ventricles in response to loss of brain mass. 


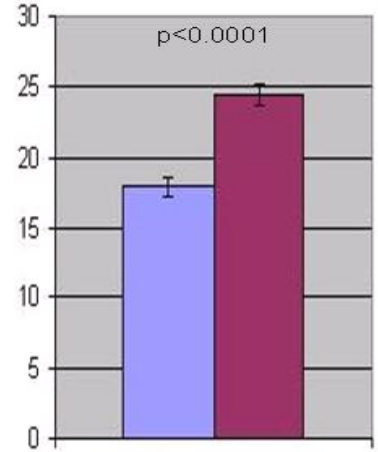

hippocampus

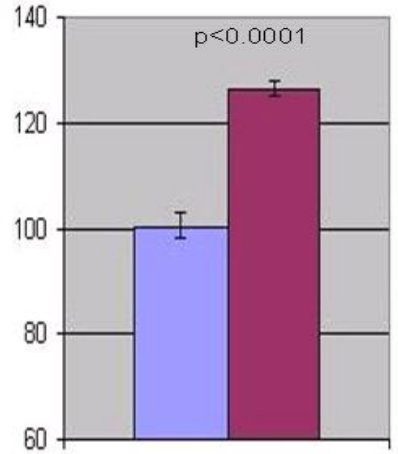

cortex

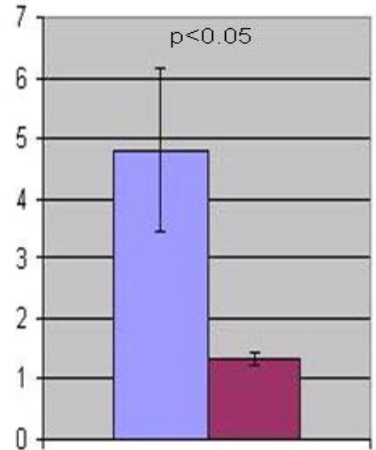

ventricle

Fig. 4. Volume comparison of three structures between transgenic and wild-type mice. Blue bar represented the average volume of $\mathrm{rTg} 4510$ mice and the red bar indicated the average volume of wild-type mice (unit: $\mathrm{mm}^{3}$ ). Statistical values between the groups are illustrated on the top of each picture.
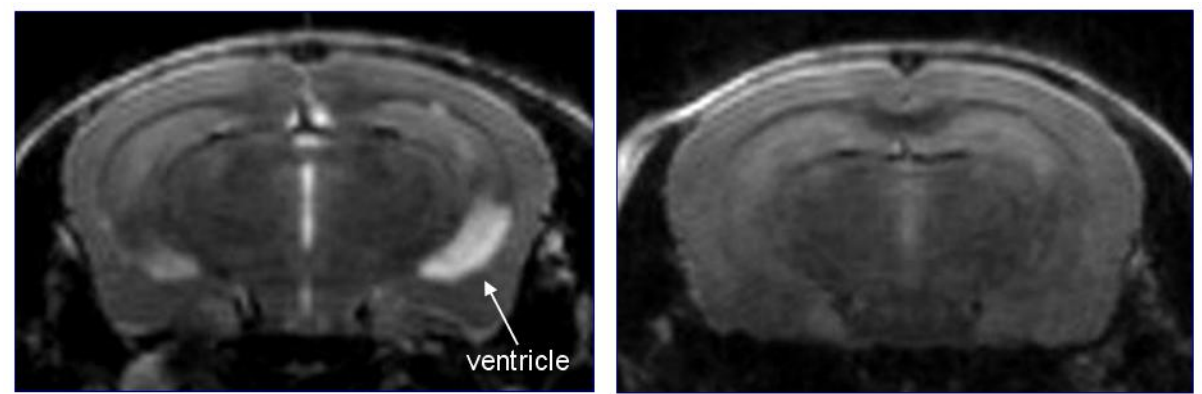

Fig. 5. MR images of a transgenic mouse (left) and a wild-type mouse (right). Ventricular dilatation (high intensity areas in the left panel) can be clearly observed in the image of transgenic mouse.

From the histopathology study, reduced cellularity in the pyramidal cell layers of the CA1 and CA2 regions and granular cell layer of the dentate gyrus was the most consistent difference between rTg4510 mice and control mice (illustrated in figure 3). Decreased cellularity of pyramidal cell layers and decreased thickness of the hippocampus were observed in 6 of $7 \mathrm{rTg} 4510$ mice, with one mouse appearing to have hippocampal thickness comparable to controls while still exhibiting neuronal loss. The thicknesses of neocortex and amygdala in the sections with hippocampus were qualitatively reduced in 3 of the $\operatorname{rTg} 4510$ mice. Four $\operatorname{Tg} 4510$ mice had minimal to mild dilatation of the ventricles. No significant cellular changes in the cerebellum were observed. These histopathology results correlated with the MRI findings in the same animals and with published reports demonstrating neuropathologic changes in the same mice [22]. 


\section{Discussion}

The present study shows that in-vivo MRI is capable of detecting cortical and hippocampal atrophy and ventricular dilatation of rTg4510 at 5.5 months of age. This time point corresponds with the age which reportedly exhibits neurodegeneration in both published reports [4] as well as in our own histopathology examination. These results suggest that noninvasive imaging can be used as a potentially translatable biomarker for progressive neuronal degeneration in this model.

In MRI analysis, TBM provides a qualitative way to characterize the location of atrophy and dilatation as a group, while the segmentation of different structures gives a quantitative way to measure the atrophy and dilatation of anatomical structures for an individual mouse. The 2 methods provide complementary information to monitor the disease progression in transgenic mice.

Future studies will include longitudinal MRI measurements in parallel with behavioral evaluation to investigate the power of MRI in detecting the disease progression at different stages. Since the rTg4510 mutant model was designed with a promoter that can be repressed with tetracycline treatment, it is possible to turn off transgene expression of tau and ask whether MRI can detect reversibility of the phenotype.

\section{References}

1. Iqbal, K., Grundke-Iqbal, I.: Alzheimer neurofibrillary degeneration: Significance, etiopathogenesis, therapeutics and prevention. J. Cell Mol. Med. 12, 38-55 (2008)

2. Bondareff, W., Mountjoy, C.Q., Roth, M., Hauser, D.L.: Neurofibrillary degeneration and neuronal loss in alzheimer's disease. Neurobiology of Aging 10(6), 709-715 (1989)

3. Duff, K., Suleman, F.: Transgenic mouse models of Alzheimer's disease: How useful have they been for therapeutic development? Briefings in Functional Genomics and Proteomics 3(1), 47-59 (2004)

4. SantaCruz, K., Lewis, J., Spires, T., et al.: Tau suppression in a neurodegenerative mouse model improves memory function. Science 309, 476-481 (2005)

5. Du, A.T., Schuff, N., Chao, L.L., Kornak, J., Jagust, W.J., Kramer, J.H., Reed, B.R., Miller, B.L., Norman, D., Chui, H.C., Weiner, M.W.: Age effects on atrophy rates of entorhinal cortex and hippocampus. Neurobiol. Aging 27, 733-740 (2006)

6. Jack Jr., C.R., Slomkowski, M., Gracon, S., Hoover, T.M., Felmlee, J.P., Stewart, K., et al.: MRI as a biomarker of disease progression in a therapeutic trial of milameline for AD. Neurology 60, 253-260 (2003)

7. McEvoy, L.K., Fennema-Notestine, C., Roddey, J.C., Hagler Jr., D.J., Holland, D., Karow, D.S., Pung, C.J., Brewer, J.B., Dale, A.M.: Alzheimer disease: quantitative structural neuroimaging for detection and prediction of clinical and structural changes in mild cognitive impairment. Radiology 251, 195-205 (2009)

8. Cohen, M.S., DuBois, R.M., Zeineh, M.M.: Rapid and effective correction of RF inhomogeneity for high field magnetic resonance imaging. Human Brain Mapping 10(4), 204 $211(2000)$

9. Yushkevich, P.A., Piven, J., Hazlett, H.C., Smith, R.G., Ho, S., Gee, J.C., Gerig, G.: Userguided 3D active contour segmentation of anatomical structures: Significantly improved efficiency and reliability. Neuroimage 31(3), 1116-1128 (2006) 
10. Caselles, V., Kimmel, R., Sapiro, G.: Geodesic active contours. International Journal of Computer Vision 22, 61-79 (1997)

11. Ashburner, J., Friston, K.J.: Morphometry. In: Frackowiak (ed.) Human Brain Function, pp. 707-725. Academic Press, London (2004)

12. Hua, X., Leow, A.D., Parikshak, N., Lee, S., Chiang, M., Toga, A.W., Jack Jr., C.R., Weiner, M.W., Thompson, P.M.: The Alzheimer's Disease Neuroimaging Initiative: Tensor-based morphometry as a neuroimaging biomarker for Alzheimer's disease: An MRI study of 676 AD, MCI, and normal subjects. NeuroImage 43(3), 458-469 (2008)

13. Styner, M., Gerig, G., Brechbuehler, C., Szekely, G.: Parametric estimate of intensity inhomogeneities applied to MRI. IEEE Transactions on Medical Imaging 19(3), 153-165 (2000)

14. Xie, Z., Farin, G.E.: Image registration using hierarchical b-splines. IEEE Transactions on Visualization \& Computer Graphics 10(1), 85-94 (2004)

15. Xie, Z., Ng, L., Gee, J.C.: Two algorithms for non-rigid image registration and their evaluation. In: Proc SPIE Medical Imaging, Image Processing (2003)

16. Thirion, J.: Image matching as a diffusion process: an analogy with Maxwell's demons. Med. Image Anal. 2(3), 243-260 (1998)

17. de Leon, M.J., George, A.E., Stylopoulos, L.A., Smith, G., Miller, D.C.: Early marker for Alzheimer's disease: the atrophic hippocampus. Lancet 2, 672-673 (1989)

18. Mouton, P.R., Martin, L.J., Calhoun, M.E., Dal Forno, G., Price, D.L.: Cognitive decline strongly correlates with cortical atrophy in Alzheimer's dementia. Neurobiology of Aging 19(5), 371-377 (1998)

19. Nestor, S.M., Rupsingh, R., Borrie, M., Smith, M., Accomazzi, V., Wells, J.L., Fogarty, J., Bartha, R.: Ventricular enlargement as a possible measure of Alzheimer's disease progression validated using the Alzheimer's disease neuroimaging initiative database. Brain 131, 2443-2454 (2008)

20. Grundman, M., Sencakova, D., Jack Jr., C.R., Petersen, R.C., Kim, H.T., Schultz, A., et al.: Brain MRI hippocampal volume and prediction of clinical status in a mild cognitive impairment trial. Journal of Molecular Neuroscience 19, 23-27 (2002)

21. Dickerson, B.C., Bakkour, A., Salat, D.H., Feczko, E., Pacheco, J., Greve, D.N., Grodstein, F., Wright, C.I., Blacker, D., Rosas, H.D., Sperling, R.A., Atri, A., Growdon, J.H., Hyman, B.T., Morris, J.C., Fischl, B., Buckner, R.L.: The cortical signature of Alzheimer's disease: regionally specific cortical thinning relates to symptom severity in very mild to mild AD dementia and is detectable in asymptomatic amyloid-positive individuals. Cereb. Cortex 19, 497-510 (2009)

22. Spires, T.L., Orne, J.D., SantaCruz, K., Pitstick, R., Carlson, G.A., Ashe, K.H., Hyman, B.T.: Region-specific dissociation of neuronal loss and neurofibrillary pathology in a mouse model of tauopathy. Am. J. Pathol. 168, 1598-1607 (2006)

23. Larner, A.J.: The Cerebellum in Alzheimer's Disease. Dement Geriatr Cogn. Disord. 8, 203-209 (1997)

24. Thomann, P.A., Santos, V.D., Essig, M., Schroder, J.: P1-299: The cerebellum in mild cognitive impairment and Alzheimer's disease: A structural MRI study. Alzheimer's and Dementia 4(4) (suppl. 1); Alzheimer's Association International Conference on Alzheimer's Disease, Alzheimer's Association International Conference on Alzheimer's Disease, p.T307 (2008)

25. Ma, Y., Smith, D., Hof, P.R., Foerster, B., Hamilton, S., Blackband, S.J., Yu, M., Benveniste, $\mathrm{H}$.: In vivo $3 \mathrm{D}$ digital atlas database of the adult $\mathrm{C} 57 \mathrm{BL} / 6 \mathrm{~J}$ mouse brain by magnetic resonance microscopy. Front. Neuroanat. 2, 1 (2008) 